\title{
Influence of randomness of buckling coefficient on the reliability index's value under fire conditions
}

\author{
Katarzyna Kubicka ${ }^{1, *}$, Urszula Radoń ${ }^{1}$ \\ ${ }^{1}$ Kielce University of Technology, Faculty of Civil Engineering and Architecture, Al. Tysiąclecia Państwa Polskiego 7, Kielce 25-314, \\ Poland
}

\begin{abstract}
The paper concerns reliability analysis of steel trusses under fire conditions with taking into account randomness of buckling coefficient, modulus of elasticity's and yield strength's reduction factors. To assess the reliability system analysis was employed. The calculations of appropriate standard deviations of random variables were realized in Mathematica program. Two types of trusses were analysed: statically determinate and indeterminate, so respectively serial and mixed system were used. The results, gotten for different levels of coefficients of variation of reduction factors were presented.
\end{abstract}

\section{Introduction}

The reliability analysis is field of interests of many scientists, not only in Poland [1-8], but all over the world [9-11]. Far fewer studies relate fire analysis of steel structures to the reliability analysis [12-18]. The following paper is concentrated on the system reliability analysis of steel trusses under fire conditions. There are two basic types of reliability systems: serial and parallel. A serial system is appropriate for structures that are statically determinate. It should be noted that for such a system a failure of one element is equivalent to the failure of the whole structure. The minimal critical set of elements (MCSE) contains only one element and the number of kinematically admissible failure mechanisms (KAFMs) is equal to the number of causative elements. The reliability of the serial system is computed according to the formula:

$$
R=\prod_{i=1}^{n} R_{i},
$$

where: $n$ is the number of the causative elements of the system and $R_{i}$ is the reliability of a single element.

Increase in the safety of the serial system can be obtained by two means, namely by increasing the safety of the weakest element of the system, or by limiting the number of elements that are linked in series.

A parallel system is suitable for some structures that are statically indeterminate. For parallel system, the structure remains reliable as long as only one element is reliable. The reliability of the parallel system is computed according to the formula:

$$
R=1-\prod_{i=1}^{n}\left(1-R_{i}\right)
$$

Generally, increasing the number of elements that are connected in parallel leads to an increase in the reliability of the whole system. However, it should be noted that when the reliability values of individual elements are high, which is the case in building structures, the strengthening effect is much less visible. Also, the addition of the fourth element actually does not produce a reliability increase. As a result, to increase the reliability of the parallel system, increasing the reliability of individual elements turns out to be a better strategy than further adding of elements.

Most statically indeterminate structures correspond to the mixed system. The basic subcategories include parallelserial and serial-parallel systems. Actually, mixed systems are usually much more complex. Statically indeterminate bar structural systems have many kinematically admissible failure mechanisms (KAFMs), in which causative elements are found. The same elements are components of more than one minimal critical set of elements (MCSE). Then, estimation of the safety of the structure is a difficult task that gets even more complex as the numbers of MCSE and causative elements increase. As a result, a simplified estimation, i.e. lower estimate of the structure safety is applied. That involves unseparating minimal critical sets of elements that have common causative elements. If MCSEs are unseparated, it can be assumed that all kinematically admissible failure mechanisms defined for a given structure are connected to one another in series. Further in the paper, the assumption holds that MCSEs are unseparated.

In the paper, to identify truss mechanisms, the spectral analysis of the linear stiffness matrix is used [19-21]. The probabilistic analysis were conducted for structures under fire design situation, permanent designed situation was not considered. The reference value of the reliability index under fire condition is equal 1.34 and it is assumed regard to [12]. 


\section{Methods of analysis}

The reliability of whole structure, independently from type of system, is function of all elements' reliabilities. To estimate reliability of $i$-th element the expected value $\left(\mu_{Z i}\right)$ and standard deviation $\left(\sigma_{Z i}\right)$ of safety margin must be computed. This is expressed as follows:

$$
\begin{gathered}
\mu_{Z_{i}}=\mu_{N_{i}}-\mu_{E f f_{i}}, \\
\sigma_{Z_{i}}=\sqrt{\sigma_{N_{i}}^{2}+\sigma_{E f f_{i}}^{2}},
\end{gathered}
$$

where: $\mu_{N i}$ and $\mu_{E f f i}$ are respectively expected values of capacity and of effect of actions for $i$-th element, $\sigma_{N i}$ and $\sigma_{E f f}$ are appropriate standard deviations. The reliability index for single element is defined as ratio of expected value of safety margin to its standard deviation:

$$
\beta_{i}=\frac{\mu_{Z_{i}}}{\sigma_{Z_{i}}}
$$

If the reliability index $\beta_{i}$ is known, it is possible to compute the probability of the element failure $\left(P_{f i}\right)$ and the reliability for a single element $\left(R_{i}\right)$ :

$$
\begin{gathered}
P_{f i}=\Phi\left(-\beta_{i}\right), \\
R_{i}=1-P_{f_{i}},
\end{gathered}
$$

where: $\Phi(\cdot)$ - the Laplace function.

Knowing reliabilities of single elements $\left(R_{i}\right)$ the reliability of whole structure $(R)$ may be estimated by using appropriate model of system. Then the probability of failure is computed as:

$$
\begin{gathered}
P_{f}=1-R, \\
\beta=-\Phi^{-1}\left(P_{f}\right) .
\end{gathered}
$$

In the following paper the probabilistic method described above was used to estimate reliability of steel trusses under fire conditions. Thermal analysis was conducted according to Eurocode in deterministic way. The probabilistic model included capacities of elements and effects of actions. Both values were assumed to have normal distribution. The coefficient of variation for effect of action was assumed to be equal $6 \%$. It should be noticed that capacity is the function of few random variables, what generates a problem with calculating standard deviation. If $f$ is the function of uncorrelated variables $X_{i}$ to $X_{m}$ and $m$ is number of variables approximate value can be gotten by using following equation [22]:

$$
\sigma \approx \sqrt{\sum_{i=\Lambda}^{m}\left(\frac{\partial f}{\partial X_{i}}\right)^{2} \sigma_{X i}^{2} .}
$$

The capacity of tension member under fire condition is defined as follows [23]:

$$
N_{t, f i, \theta}=A \cdot f_{y} \cdot k_{y, \theta},
$$

where: $A$ - area of cross-section, $f_{y}$-yield strength, $k_{y, \theta^{-}}$reduction factor for effective yield strength. All these variables are random. So the standard deviation computed using equation (10) is equal:

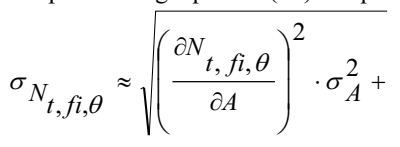

$+\overline{\left(\frac{\partial N_{t, f i, \theta}}{\partial f_{y}}\right)^{2} \cdot \sigma_{f_{y}}^{2}+\left(\frac{\partial N_{t, f i, \theta}}{\partial k_{y, \theta}}\right)^{2} \cdot \sigma_{k, \theta}^{2}}$

what gives following formula:

$$
\begin{aligned}
& \sigma_{N_{t, f i, \theta}} \approx \sqrt{f_{y}^{2} \cdot k_{y, \theta}^{2} \cdot \sigma_{A}^{2}+} \\
& +A^{2} \cdot k_{y, \theta}^{2} \cdot \sigma_{f_{y}}^{2}+A^{2} \cdot f_{y}^{2} \cdot \sigma_{k, \theta}^{2}
\end{aligned}
$$

The values of coefficient of variation of random variables for element in tension, that were assumed to calculation, are presented in table 1. The choice of coefficient of variation's value for reduction factor $k_{y, \theta}$ is problematic. According to [24] it is recommended to assume it as equal $20 \%$. But the calculation, which were conducted by authors of article indicated that it seems to be too big value. So other values were analysed $(5 \%$ and $7 \%$ ). The situation with deterministic character of reduction factor for yield strength $\left(k_{y, \theta}=0\right)$ was also analysed.

Table 1. Coefficient of variation for random variables of elements in tension.

\begin{tabular}{|l|l|}
\hline Random variable & Coefficient of variation \\
\hline Area of cross section $(A)$ & $6 \%$ \\
\hline Yield strength $(f y)$ & $8 \%$ \\
\hline $\begin{array}{l}\text { Reduction factor for yield } \\
\text { strength }\left(k_{y}, \theta\right)\end{array}$ & $0 \%, 5 \%, 7 \%$ or $20 \%$ \\
\hline
\end{tabular}

For the elements that are compressed the capacity under fire conditions is computed as [23]:

$$
N_{b, f i, \theta}=\chi_{f i} \cdot A \cdot k_{y, \theta} \cdot f_{y},
$$


where: $A, k_{y, \theta}$ i $f_{y}$ are defined as in $(11), \chi_{f i}$ is the buckling coefficient, which is the function of few variables. It is defined as [23]:

$$
\chi_{f i}=\frac{1}{\varphi_{\theta}+\sqrt{\varphi_{\theta}^{2}-{\overline{\lambda_{\theta}}}^{2}}},
$$

where:

$$
\varphi_{\theta}=\frac{1}{2}\left(1+\alpha{\overline{\lambda_{\theta}}}+{\overline{\lambda_{\theta}}}^{2}\right)
$$

and

$$
\alpha=0.65 \sqrt{\frac{235}{f_{y}}} .
$$

The non-dimensional slenderness $\overline{\lambda_{\theta}}$ for the temperature of element $\theta$, is given by [23]:

$$
\overline{\lambda_{\theta}}=\bar{\lambda} \cdot\left(\frac{k_{y, \theta}}{k_{E, \theta}}\right)^{0.5},
$$

where: $k_{y, \theta}$ and $k_{E, \theta}$ are respectively reduction factors for yield strength and for modulus of elasticity, $\bar{\lambda}$ is the slenderness in normal design conditions and is defined as [23]:

$$
\bar{\lambda}=\sqrt{\frac{A \cdot f_{y}}{N_{c r}}}
$$

The critical force $N_{c r}$, described by equation (20), depends on: modulus of elasticity $(E)$, minimum moment of inertia $\left(I_{y}\right)$ and the length of element $(L)$.

$$
N_{c r}=\pi^{2} \frac{E J_{\min }}{L^{2}} .
$$

All variables, which are used to compute coefficient of buckling, were treated as probabilistic. The assumed coefficients of variation for random variables of compressed elements are presented in table 2. For reduction factors of modulus of elasticity and yield strength different values were considered. To compute value of buckling coefficient $(\chi)$ few other coefficient, defined by equations (16-20) have to be determined. All of them depend on random variables enumerated in table 2 , so they also have probabilistic character.

Table 2. Coefficient of variation for random variables of elements in compression.

\begin{tabular}{|l|l|}
\hline Random variable & $\begin{array}{l}\text { Coefficient of } \\
\text { variation }\end{array}$ \\
\hline Area of cross section $(A)$ & $6 \%$ \\
\hline Yield strength $(f y)$ & $8 \%$ \\
\hline $\begin{array}{l}\text { Reduction factor for yield } \\
\text { strength }\left(k_{y, \theta}\right)\end{array}$ & $0 \%, 5 \%, 7 \%$ or $20 \%$ \\
\hline Modulus of elasticity $(E)$ & $5 \%$ \\
\hline $\begin{array}{l}\text { Reduction factor for modulus of } \\
\text { elasticity }\left(k_{E, \theta}\right)\end{array}$ & $0 \%, 5 \%, 7 \%$ or $20 \%$ \\
\hline Minimum moment inertia $\left(J_{\min }\right)$ & $6 \%$ \\
\hline Length of element $(L)$ & $6 \%$ \\
\hline
\end{tabular}

The standard deviations were computed in two steps. First the standard deviation of coefficient of buckling was computed, as it is also function of few variables. According to equation (10) it could be written:

$$
\begin{aligned}
& \sigma_{\chi_{f i}} \approx \sqrt{\left(\frac{\partial \chi_{f i}}{\partial A}\right)^{2} \cdot \sigma_{A}^{2}+} \\
& +\frac{\left(\frac{\partial \chi_{f i}}{\partial f_{y}}\right)^{2} \cdot \sigma_{f_{y}}^{2}+\left(\frac{\partial \chi_{f i}}{\partial k_{y, \theta}}\right)^{2} \cdot \sigma_{k}^{2} y, \theta}{\left(\frac{\partial \chi_{f i}}{\partial k_{E, \theta}}\right)^{2} \cdot \sigma_{k}^{2}+\left(\frac{\partial \chi_{f i}}{\partial E}\right)^{2} \cdot \sigma_{E}^{2}} \\
& +\left(\frac{\partial \chi_{f i}}{\partial J_{\min }}\right)^{2} \cdot \sigma_{J_{\min }}^{2}+\left(\frac{\partial \chi_{f i}}{\partial L}\right)^{2} \cdot \sigma_{L}^{2}
\end{aligned}
$$

Then the standard deviation for capacity could be computed according to following general formula:

$$
\frac{\sigma_{N_{b, f i, \theta}} \approx \sqrt{\left(\frac{\partial N_{b, f i, \theta}}{\partial \chi_{f i}}\right)^{2} \cdot \sigma_{\chi_{f i}}^{2}}}{+\left(\frac{\partial N_{b, f i, \theta}}{\partial A}\right)^{2} \cdot \sigma_{A}^{2}+\left(\frac{\partial N_{b, f i, \theta}}{\partial f_{y}}\right)^{2} \cdot \sigma_{f y}^{2}}
$$

The computations of standard deviations according to equations (14) and (15) were performed in Mathematica program. As the outcome was extremely expanded it is not possible to present it in the article.

In the following part of paper two trusses were considered: statically determinate and indeterminate. The serial system is appropriate for the first type of structure and for the second one - mixed system, what created necessity to find KAFMs. This task was realized by using Mathematica module [19-21]. 


\section{Example of analysis}

Using methods described above, the statically determinate steel truss, shown in the figure 1 , was analysed. The only load was the dead load $p=3 \mathrm{kN} / \mathrm{m}$ applied to the top chord, and it was converted to concentrated forces in the nodes. All the elements were assumed to be heated from each side. Spray-applied mineral fibre with the thickness of $1.5 \mathrm{~cm}$ was assumed as an insulation. This material is characterized by the following parameters: density $\rho_{\mathrm{p}}=800 \mathrm{~kg} / \mathrm{m}^{3}$, specific heat $\mathrm{c}_{\mathrm{p}}=1700 \mathrm{~J} /(\mathrm{kgK})$, thermal conductivity $\lambda_{\mathrm{p}}=0.2$ $\mathrm{W} /(\mathrm{mK})$. In order to simplify the analysis, a number of assumptions were adopted in the study. The truss bars are treated as ideal rectilinear elements that do not bear initial material or geometric imperfections. Axial forces are determined without taking into account II order effects that result from additional shortening due to bending. At the normal temperature of the structure in service, i.e. at the start of the fire, the value of the initial amplitude of the element depends on the bar crosssection, buckling direction and the ratios of the section dimensions. At the temperatures that hold as the fire progresses, the influence of imperfection changes. At the instant of the limit state, this influence is set at the same level for all section types and buckling directions. The assumptions made above and the application of the buckling criterion in accordance with the Eurocode leads to the fact that the computed reliability index is inherently marred by a certain error.

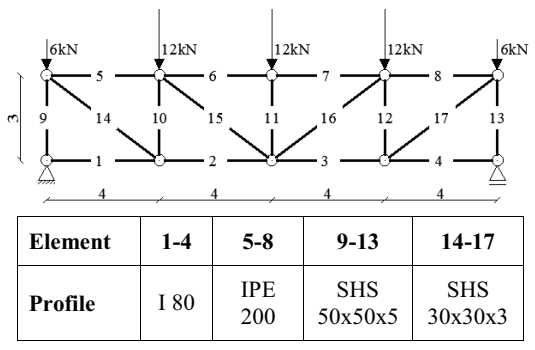

Fig. 1. Statically determinate steel truss.

To obtain effects of action FEM analysis with using MES3D program was conducted. The values of capacity of each element were gotten by using files of Mathematica program, which were created by authors of article. In this same program appropriate standard deviations were computed. The coefficient of variation for effect of action was assumed to be equal $6 \%$. The biggest problem was connected with the choice of level of coefficient of variation. According to [24] this value for reduction factor $k_{y}$ may be assumed to be equal $20 \%$. As it was mentioned above, also other values were examined.

The reliability analysis was conducted using spreadsheet of Excel. As the structure (Fig.1) is statically determinate, it corresponds to serial system. So, according to equation (1) the reliability of whole truss is the product of all elements' reliabilities. The scheme of reliability system with number of minimal critical set of elements (MCSE) is shown in the figure 2.

$$
\begin{gathered}
\begin{array}{l}
\text { I KAFM } \\
\text { (17MCSE) }
\end{array} \\
1-2-3-4-5-6-7-8-9-10-11-12-13-14-15-16-17
\end{gathered}
$$

Fig. 2. Kinematically admissible failure mechanism for statically determinate steel truss.

The results gotten for different values of reduction factors' coefficient of variation are shown in figure 7 . The reference value of reliability index is marked by dotted line. The curve $v \mathrm{ky}=0 \%, v \mathrm{kE}=0 \%$ corresponds to situation, when both reduction factors (for yield strength and modulus of elasticity) were deterministic. So, according to equations (15-20), also coefficient of buckling was deterministic. The first attempts to take into account probabilistic characteristic of those values are represented by curves $v \mathrm{ky}=20 \%, v \mathrm{kE}=20 \%$ and $v \mathrm{ky}=20 \%, v \mathrm{kE}=0 \%$. In the first case both factors were probabilistic, in the second one only reduction factors for yield strength. The received values of reliability indices seems to be too low. Before five minutes of fire duration the limit state function is exceeded. Such situation suggests that coefficient of variation for reduction factors at the level equal $20 \%$ may be overestimated. So the values of $5 \%$ and $7 \%$ in three different combinations were examined (Fig.7).

The similar analysis was conducted for statically indeterminate truss, shown in the figure 3 . The basic assumptions were the same as in the case of statically determinate structure. For previous analysed structure serial system was appropriate, what means that the whole system is reliable as long as each single elements' capacity is not exceeded. For such a case there is only one kinematically admissible failure mechanism (Fig.2) The element with the biggest effort decides about failure of whole structure. In the case of statically indeterminate structures situation is complety different. Such structures usually correspond to mixed system and they may be still safe in spite of failure of single element. To estimate reliability of statically indeterminate structure the appropriate kinematically admissible mechanisms must be identified. For analysed truss there are four types of KAFMs. All of them are presented in figure 4. As the analysed structure is statically indeterminate the effect of action was not constant in successive minutes of analysis. What is more after 25 minutes of fire duration the buckling resistance of extreme cross braces (elements 18,21) was exceeded, causing the change of static scheme. So analysis for 30 minute was conducted for the truss shown in the figure 5. For this structure three types of kinematically admissible failure mechanism were identified. They are presented in figure 6 . Because of changes described above FEM analysis in MES3D was conducted for structure with scheme presented in figure 3 until $25^{\text {th }}$ minute of fire duration. In 
the further minutes of analysis the buckled cross-braces were removed from stiffness matrix, so the analysis was conducted for structure with scheme presented in figure 5 .

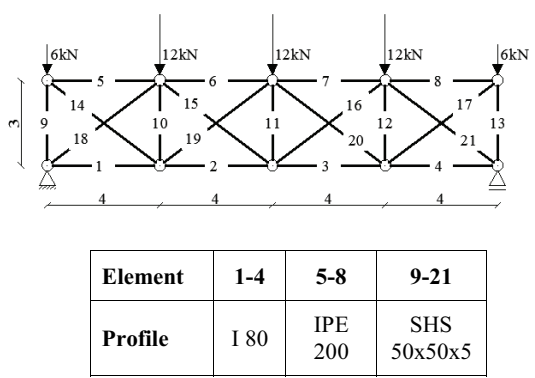

Fig. 3. Statically indeterminate steel truss.
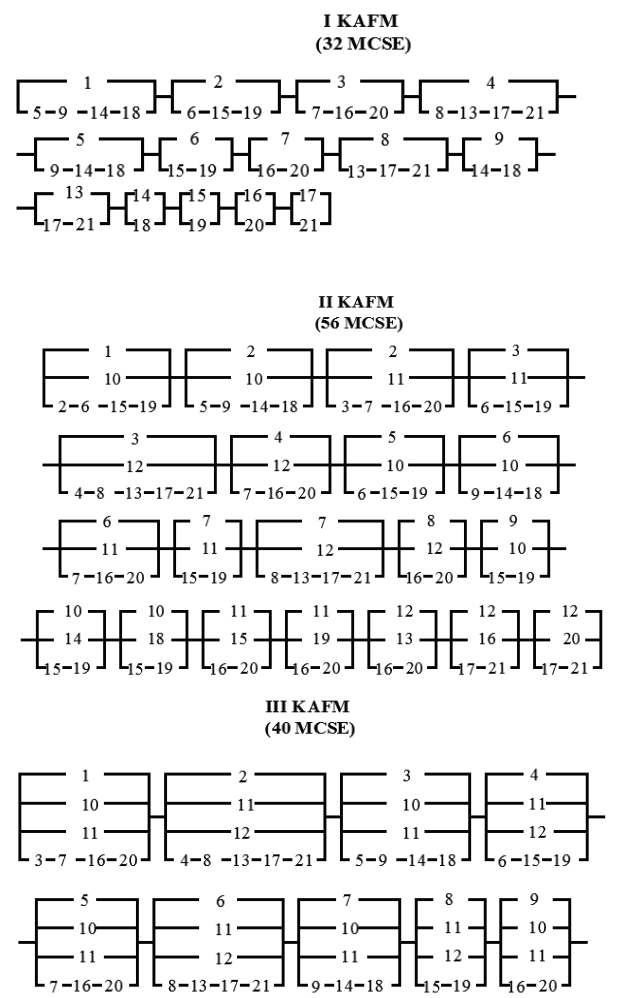

$-\left[\begin{array}{c}10 \\ 11 \\ -14 \\ 16-20\end{array}\right]\left[\begin{array}{c}10 \\ 11 \\ -18 \\ 16-20\end{array}\right]\left[\begin{array}{c}11 \\ -12 \\ -13 \\ 15-19\end{array}\right]\left[\begin{array}{c}11 \\ 12 \\ -15 \\ 17-21\end{array}\right]\left[\begin{array}{l}11 \\ 12 \\ 19 \\ L_{17-21}\end{array}\right]$
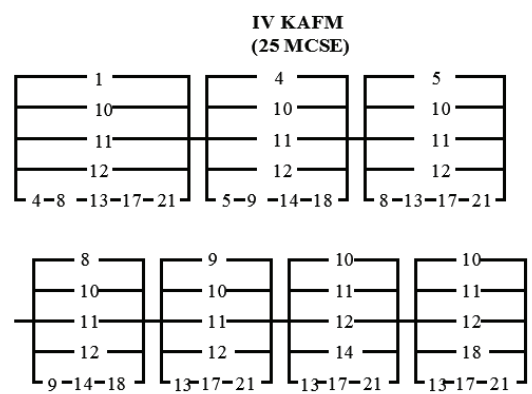

Fig. 4. Kinematically admissible failure mechanisms for indeterminate truss.

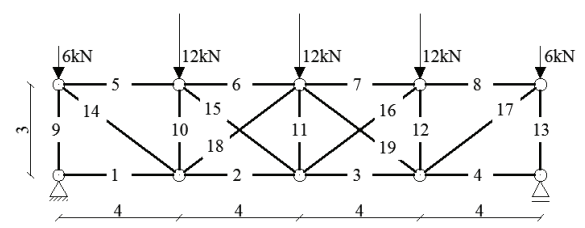

Fig. 5. Kinematically admissible failure mechanisms for indeterminate truss.

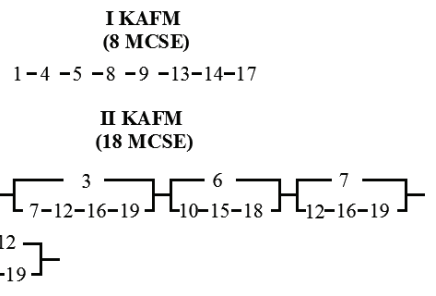

\section{KAFM}

(25 MCSE)

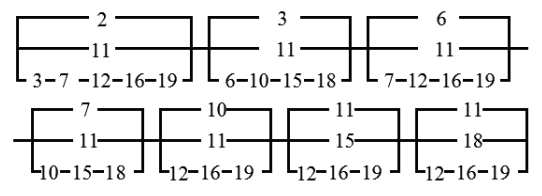

Fig. 6. Kinematically admissible failure mechanisms for indeterminate truss 


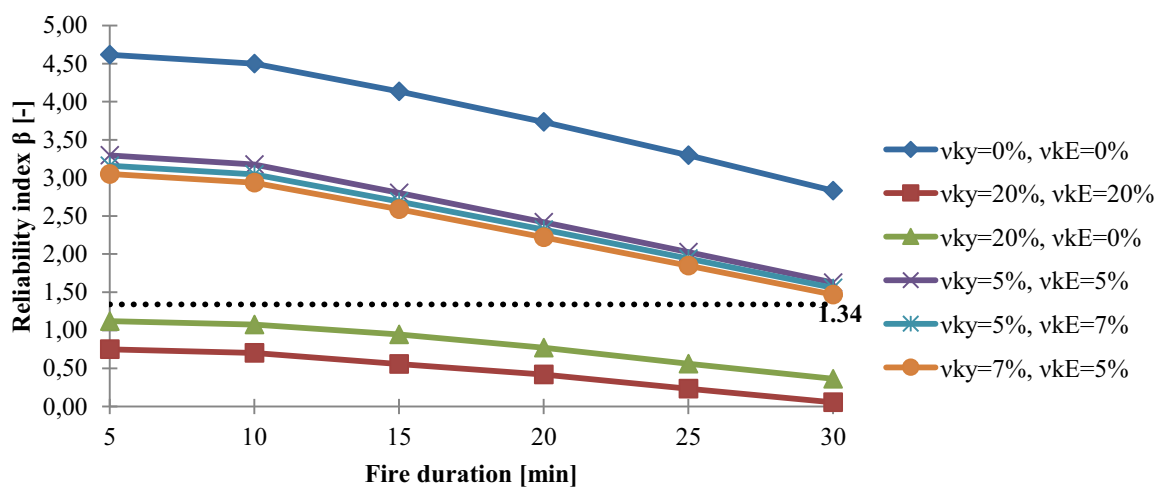

Fig. 7. Monitoring of reliability index of statically determinate steel truss under fire conditions for different values of reduction factors' coefficients of variation.

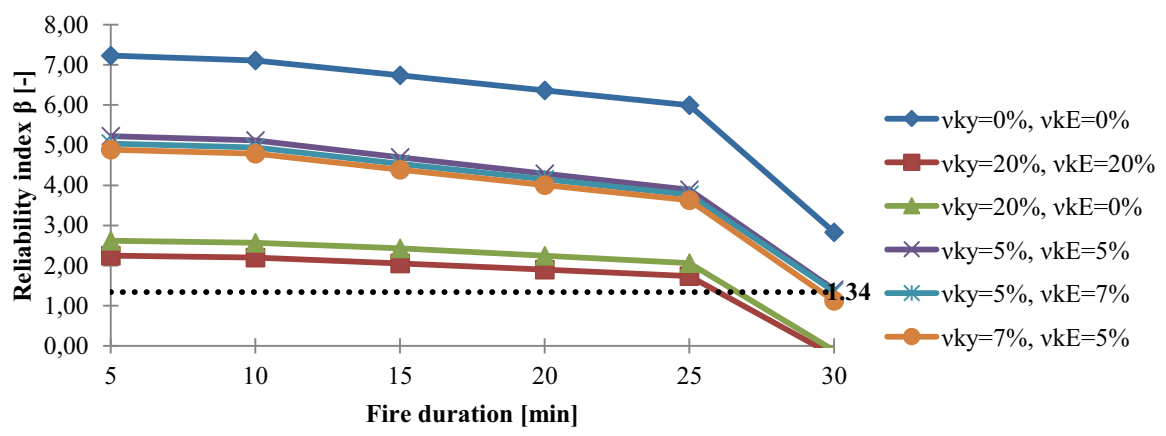

Fig. 8. Monitoring of reliability index of statically indeterminate steel truss under fire conditions for different values of reduction factors' coefficients of variation.

The results of reliability analysis for statically indeterminate truss under fire conditions are shown in the figure 8 . It should be noticed that the change of static scheme cause rapid decrease of reliability index. When coefficient of buckling is assumed to be probabilistic value, regardless of values of coefficients of variation, the limit state function is exceeded in $30^{\text {th }}$ minute. When coefficient of buckling is treated as deterministic then results indicates that structure is safety, because in $30^{\text {th }}$ reliability index is still much more higher then reference value.

\section{Conclusions}

The aim of the paper was to underline the meaning of probabilistic model definition in assessing the reliability of structure under fire conditions. Particularly the character of buckling coefficient (probabilistic or deterministic) was considered. To realize this task two theoretical trusses were analysed. To simplify analysis plenty assumptions were made, so conclusions presented below are strongly connected with them.
The analysis of graphs, presented in the figure 7 and 8 , indicates that taking into account randomness of reduction factors and buckling coefficient have a significant meaning. When these values are treated as deterministic the results are overstated. Nevertheless, the appropriate choice of coefficients of variation pose a huge problem. There is the lack of appropriate data in the literature and presented analysis are not wide enough to draw conclusions. On the assumption that coefficient of variation for yield strength is equal $20 \%$ the obtained reliability indices seems to be too low (Fig. 7,8). In such situation for statically determinate structure the limit state function is exceeded from the beginning of analysis (Fig.7). With the assumption that modulus of elasticity and its reduction factor are deterministic values the results are slightly higher, but still seem to be too low. The same conclusion may be drawn for the statically determinate truss. The analysis for other values of coefficients of variation $(5 \%, 7 \%)$ in different combination indicated that the coefficient of variation for yield strength has greater influence on the reliability 
index then the coefficient of variation for modulus of elasticity. It should be underlined that proposed coefficients of variation should not be treated as reference. The problem of proper values needs wider discussion.

\section{References}

1. A. Biegus, Probabilistic analysis of steel structures PWN, Warsaw-Wroclaw (1977) (in Polish).

2. P. Sorn, J. Górski, J. Przewłócki, Archives of Civil Engineering, 61 (2), 99-123 (2015)

3. A. Nowak, K. R. Collins, Reliability of structures. 2nd edition, (CRC Press. Taylor and Francis Group, New York, 2013)

4. R. Stocki, K. Kolanek, M. Jendo, M. Kleiber, Computers and Structures 79, 2235-2247 (2001).

5. Z. Kowal, Archives of Civil and Mechanical Engineering XI (1), 115-133 (2011).

6. A. Dudzik, U. Radoń, Advances in Mechanics: Theoretical, Computational and Interdisciplinary Issues, Taylor \& Francis Group, London, 163-166 (2016)

7. A. Dudzik, IOP Conference Series: Materials Science and Engineering 245 (2017)

8. A. Dudzik, U. Radoń, Archives of Civil Engineering LXI (3), 133-147 (2015)

9. R. Melchers, Structural reliability analysis and prediction. 2nd edition (John Wiley \& Sons, New York, 2002)

10. O. Ditlevsens, H. Madsen, Structural reliability methods (Wiley, 1996)

11. P. Thoft-Christensen, M. Baker, Structural reliability theory and its applications (SpringerVerlag, Berlin Heidelberg New York, 1982)

12. M. Maślak, Eighth International Conference on Advances in Steel Structures (Lisbon ,2015).

13. K. Kubicka, U. Radoń, W. Szaniec, U. Pawlak, IOP Conference Series: Materials Science and Engineering 245 (2017)

14. K. Kubicka, U. Radoń, W. Szaniec, IOP Conference Series: Materials Science and Engineering $\mathbf{2 4 5}$ (2017)

15. K. Kubicka, U. Radoń, Archives of Civil Engineering 61 (4), 141-154 (2015)

16. J. Králik, T. Varga, Proceedings of the European Safety and Reliability Conference 2006, ESREL 2006, Safety and Reliability for Managing Risk, 2081-2086 (Estoril, Portugal, 2006)

17. Q. Guo, A. Jeffers, Application of Structural Fire Engineering (Prague ,2013).

18. G. De Sanctics, M. H. Faber, M. Fontana, Fire safety science-proceedings of the eleventh international symposium, 996-1009 (2014).

19. K. Dems, J. Turant, Bulletin of the Polish Academy of Sciences: Technical Sciences 59 (1), 27-32 (2011)

20. W. Gilewski, J. Kłosowska, P. Obara, Advances in Mechanics: Theoretical, Computational and
Interdisciplinary Issues, Taylor \& Francis Group, London 191-195 (2016)

21. J. Kłosowska, P. Obara, J. Turant, IOP Conference Series: Materials Science and Engineering $\mathbf{2 4 5}$ (2017)

22. W.W. Bołotin Statistical methods in structural mechanics (1968)

23. PN-EN-1993-1-2. Eurocode 3: Design of steel structures-Part 1-2: General rules: Structural fire design

24. M. Holicky, A. Materna, G. Sedlacek, J.-B. Schleich, A. Arteaga, L. Sanpaolesi, T. Vrouwenvelder, I. Kovse, H. Gulvanessian Implementation of Eurocodes. Handbook. Design of buildings for the fire situation (Luxembourg $10 / 2005)$ 